$\xi$

\title{
An Approach to Estimate the Outstanding Loss Reserve of the Non-Life Insurer Under Solvency- II Regime
}

\author{
Ashiq Mohd Ilyas ${ }^{1 *}$, S. Rajasekaran ${ }^{2}$ \\ ${ }^{1,2}$ Department of Mathematics and Actuarial Science, \\ B.S. Abdur Rahman Crescent Institute of Science and Technology \\ *Corresponding author E-mail:nashiq.ilyas@gmail.com
}

\begin{abstract}
This paper studies the reserve risk estimation requirement under the Solvency-II regime that came into effect in the European insurance sector in January 2016. In particular, it shows how the outstanding loss of a non-life insurer can be estimated under this regime. This regime totally replaces the traditional approaches of providing standard deviations of the liabilities over their full run-off. The requirement under this regime is that each risk shall be calibrated using a value-at-risk measure with 99.5 percentile confidence level over a single period. In connection with this, a bootstrap framework is used to estimate the uncertainty of loss reserve over the single period time horizon. Two process distributions are used namely Over-dispersed Poisson and Gamma in two separate bootstraps to estimate the uncertainty of loss reserve. Further, a comparison is established in the estimated results and it is found that Over-dispersed Poisson process distribution produces lower prediction errors than the gamma process distribution.
\end{abstract}

Keywords: Bootstrap, Chain Ladder Model, Claims Development Result, Claims Reserve, Solvency-II

\section{Introduction}

The Solvency-II regime aims to set up improved risk sensitive solvency requirements for insurers functioning in Europe. It proposed its roots in the insurance business with European Parliament and the council directive [1]. It practically came into effect from January 2016. This regime pursuit to provide the fair value of capital required to meet the technical provision of the insurer. The technical provision is the major and most important portion of liabilities on balance sheet of the insurer. The reserve risk arises when the technical provision is insufficient to cover the claims whether unsettled, un-expired or not reported to the insurer. Therefore an insurer has to estimate reserve as accurately as possible to mitigate the reserve risk. Under solvency-II, each risk shall be calibrated using a value-at-risk measure with 99.5 percentile confidence level over 12 month period [2]. It replaces the ultimate reserve risk view with single period reserve risk view. In simpler words, it corresponds to the situation when the current reserve is insufficient to cover their run-off over the period of oneyear time horizon. Solvency-II totally replaces the traditional approaches of providing standard deviations of the liabilities over their runoff. Moreover, the reserve risk ignores all complications like credit risk on reinsurance, operational risk loading under this regime. This characteristic shifts the risk profile only to the profitability of reserve held over a single year period known as the Claims Development Result (CDR). The single year CDR is the expected ultimate cost of claims at opening less the expected cost of claims after one year. The CDR for the year $i \in\{1, \ldots, I\}$ in accounting year $\{I, I+1\}$ according to definition 2.15 given in [3] can be defined as:
$\operatorname{CDR}(I+1)=E\left(C_{i j} \mid D_{I}\right)-E\left(C_{i j} \mid D_{I+1}\right)$

The reserve risk can be computed either by the standard formula of solvency-II or alternatively by the approved internal models. The Chain Ladder (CL) model is one of the permitted internal models to estimate outstanding loss reserve under solvency-II [2]. It is the benchmark for most of the reserving models in the literature. The CDR concept can be used in the CL method to fulfil the single-year horizon uncertainty requirement. However, it does not generate $99.5 \%$ percentile as required under solvency-II. The estimation of the prediction uncertainty in CL model provides great insight into the future claims development. However, the full predictive distribution of reserve estimates will provide information about cash flows as well as risk measures. With this attention, this study use bootstrap technique to produce the full predictive distribution of estimates and the associated uncertainty according to the requirements of the solvency-II.

This paper is organized as follows. The section 2 reviews the related literature and highlights the aim of the study. Section 3 discusses the incorporation CDR concept in CL model and issue underlying this method under the solvency-II regime. Section 4 illustrates a Bootstrap algorithm to produce an empirical distribution of CDR. In addition, reflect final results and the associated findings. The final section concludes.

\section{Literature Review}

The most popular run-off method in practice usually used by the actuaries for claim reserving is the CL model. Earlier this was referred as a purely deterministic algorithm for the estimation of claim reserve. However, Mack [4] introduced the distribution-free CL model and derived a formula for estimation errors. This be- 
came the foundation stone for the quantification of prediction uncertainties, which cause reserve risk. Presently wide variety of stochastic reserving methods supports CL model (for an overview see [5].Although many researchers have given different approaches to derive an estimate for estimation error [see e.g. 6, 7].

However, all these approaches are very similar to one another. The single year CDR made CL model best fit for the solvency II one year horizon requirement.

There are mainly three concepts behind the adaptation of CDR [8] The first one is the elusive nature of the ultimate unpaid loss. The second one is the effect of information about claim data obtained over one year. The last one is the actuarial methodology used to predict the best estimate one year later. Merz \& Wüthrich [3] incorporated this concept in chain ladder model to estimate reserve uncertainty over the one-year time horizon. However, under solvency-II, each risk shall be calibrated using a value-at-risk measure with 99.5 percentile confidence level over 12 month period (Article 104 of solvency II directive). There are other studies that deal with one- year reserve risk estimation however, this question is not addressed by any of these studies [see e.g. 9, 10]. This study argues that bootstrap is the most appropriate method to resolve this issue. The bootstrap method is first proposed by Efron [11] This approach is widely used in the actuarial literature [see e.g. 9 , $12,13]$.These researchers combined the bootstrap concept with the CL model.

Our study aims to estimate the outstanding loss and the related prediction errors under the solvency-II requirement. We use Bootstrapping to produce a predictive distribution of the CDR from which 99.5 percentile of CDR can be estimated. To produce the distribution the Gamma and over-dispersed-Poisson distribution are used as process distributions instead of commonly used lognormal distribution. In addition, we case study the bootstrapping method by using the real claims data from [13]. Moreover, a comparison is drawn between prediction uncertainties of gamma bootstraps and over-dispersed Poisson bootstrap.

\section{Case Study: Chain Ladder Model}

The CL model is the base for calculating the best estimate in most of reserving methods. The estimation of best estimate is mainly used to estimate the outstanding claims namely Incurred But Not Reported (IBNR) claims. These are claims that take years to emerge due to the intricate damage, long legal procedures or strenuous estimation of the claim amount. Therefore an insurer has to structure reserve for future claims arising from written contracts as well as for outstanding claims. For the estimation of the outstanding claims by CL model, we can use the paid claim data or incurred claim data.

Consider a family of positive random variable $\mathrm{X}_{\mathrm{ij}}$ denote the individual claim amounts, where $i \in(1, \ldots, \mathrm{I})$ denote accident year and $j \in(1, \ldots, J)$ denote development year. Then the cumulative claim amount can be defined as $\mathrm{C}_{\mathrm{ij}}=\sum_{\mathrm{k}=1}^{\mathrm{J}} \mathrm{X}_{\mathrm{ik}}$. The outstanding claim reserve for accident year $\mathrm{i}$ can be defined as

$\mathrm{R}_{\mathrm{i}}=\mathrm{C}_{\mathrm{i}}-\mathrm{C}_{\mathrm{i}, \mathrm{J}-\mathrm{i}+1} 1 \leq \mathrm{i} \leq \mathrm{J}$

In standard CL model reserve risk is measured by mean square error without assuming any distribution. We refer the time series version assumption of chain ladder model as these assumptions are stronger than the assumption laid down in basic Mack's model So, in order to predict outstanding claims, the following assumptions have to be carried out.

1. The cumulative claim amounts $\mathrm{C}_{\mathrm{ij}}$ are independent.

2. There exist development factors $\left(f_{i}>0\right)_{i} \geq 0$ for $1 \leq j \leq J$

$$
E\left[C_{i j} \mid C_{i, j-1}\right]=f_{j-1} C_{i, j-1}
$$

3. There exist variance parameters $\left(\sigma_{i} \geq 0\right)_{i} \geq 0$ for $1 \leq \mathrm{j} \leq \mathrm{J}$

$$
\operatorname{var}\left(C_{i j} \mid C_{i, j-1}\right)=\sigma_{j-1}^{2} C_{i, j-1}
$$

The estimation of development factors that are the unbiased and uncorrelated estimator of $f_{k}$ is defined as

$$
\hat{\mathrm{f}}_{\mathrm{k}}=\frac{\sum_{\mathrm{j}=1}^{\mathrm{I}-\mathrm{k}} \mathrm{C}_{\mathrm{i}, \mathrm{k}+1}}{\sum_{\mathrm{j}=1}^{\mathrm{I}-\mathrm{j}-1} \mathrm{C}_{\mathrm{ik}}} \quad 1 \leq \mathrm{k} \leq \mathrm{I}-1
$$

Therefore the estimated ultimate claim amount can be defined by

$$
\hat{\mathrm{C}}_{\mathrm{iJ}}=\mathrm{C}_{\mathrm{i}, \mathrm{I}-\mathrm{i}} \prod_{\mathrm{k}=\mathrm{I}-\mathrm{i}}^{\mathrm{J}-1} \hat{\mathrm{f}_{\mathrm{k}}}
$$

This estimator is used as a predictor for future claims. The predictive uncertainty that is the mean square error prediction of the ultimate claim estimator is defined in [4] as

$$
\begin{aligned}
& \operatorname{MSEP}\left(\hat{\mathrm{C}}_{\mathrm{ij}}\right)=\operatorname{E}\left[\left(\mathrm{C}_{\mathrm{ij}}-\hat{\mathrm{C}}_{\mathrm{ij}}\right)^{2} \mid \mathrm{D}\right] \\
& \operatorname{MSEP}\left(\hat{\mathrm{C}}_{\mathrm{ij}}\right)=\operatorname{Var}\left(\mathrm{C}_{\mathrm{ij}} \mid \mathrm{D}\right)+\mathrm{E}\left(\left(\mathrm{C}_{\mathrm{ij}} \mid \mathrm{D}\right)-\hat{\mathrm{C}}_{\mathrm{ij}}\right)^{2}
\end{aligned}
$$

The MSEP formula of basic CL is projecting the total uncertainty of loss reserve over the entire runoff. However, under solvency-II, the actuaries need to quantify prediction uncertainties over next accounting year. It means require a dynamic view of prediction uncertainties.

For this reason, the derivation of 12 months CDR for distribution free CL model by Merz \& Wüthrich [3] got a lot of attention in reserving under Solvency II regime. The primary objective of this study is to measure the uncertainty associated with the reevaluation of the best estimate between the time I to time I+1.The CDR analyze the reserve uncertainties over a 12-month horizon. For the proof of the CDR and other results, which are used thereafter in this section refer to [3].The observed CDR for accident year in next accounting year when the chain ladder factors are unknown is defined by

$$
\sum_{i=1}^{I} \hat{C D R_{i}}(I+1)
$$

The Mean Square Error Prediction of claims development is derived in section 3 of [3]. For a single accounting year, the conditional mean square error of prediction of the observed CDR by 0 given information $D_{I}$ is defined by

$$
\operatorname{MSEP}_{\mathrm{CDR}(\mathrm{I}+1) \mid \mathrm{D}_{\mathrm{I}}}(0)=\mathrm{E}\left[\left(\hat{\mathrm{C}}_{\mathrm{ij}}^{\mathrm{I}}-\hat{\mathrm{C}}_{\mathrm{ij}}^{\mathrm{I}+1}\right)^{2} \mid \mathrm{D}_{\mathrm{I}}\right]
$$

The CL model is employed to CL obtain from [13] to estimate the claims reserve and the related uncertainty. The subjective estimation results are enclosed in Table 1 . The mean square prediction error of the observed CDR for single accounting year is estimated by using Equation 8 .

Table 1: Reserve estimate by CL model

\begin{tabular}{|c|c|c|c|c|c|}
\hline Year & $\begin{array}{c}\text { Mean } \\
\text { Ultimate } \\
\text { loss }\end{array}$ & $\begin{array}{c}\text { Mean } \\
\text { IBNR }\end{array}$ & $\begin{array}{c}\text { Mack. } \\
\text { msep }^{1 / 2}\end{array}$ & $\begin{array}{c}\text { CV } \\
\text { IBNR }\end{array}$ & $\begin{array}{c}\text { CDR } \\
\text { msep }^{1 / 2}\end{array}$ \\
\hline 1 & 7518 & 0 & 0 & 0 & 0 \\
\hline 2 & 9470 & 15.1 & 2.09 & 0.1 & 2.1 \\
\hline 3 & 13223 & 62.4 & 10.95 & 0.2 & 10.7 \\
\hline 4 & 9247 & 143.7 & 37.59 & 0.3 & 36.8 \\
\hline
\end{tabular}




\begin{tabular}{|c|c|c|c|c|c|}
\hline 5 & 7804 & 218.2 & 43.49 & 0.2 & 28.4 \\
\hline 6 & 7734 & 399.2 & 92.31 & 0.2 & 82.0 \\
\hline 7 & 8614 & 822.7 & 164.92 & 0.2 & 133.4 \\
\hline 8 & 11093 & 1842 & 378.65 & 0.2 & 328.4 \\
\hline 9 & 10118 & 4056 & 980.31 & 0.2 & 913.7 \\
\hline 10 & 9847 & 8764 & 1928.5 & 0.22 & 1673.6 \\
\hline $\begin{array}{c}\text { To- } \\
\text { tal }\end{array}$ & 94668 & $\begin{array}{c}1632 \\
3\end{array}$ & 2285 & 0.1 & 2016 \\
\hline
\end{tabular}

$\operatorname{MSEP}\left(\hat{\mathrm{C}}_{\mathrm{ij}}\right) \cong \varphi \mu_{\mathrm{ij}}^{\delta}+\mu_{\mathrm{ij}}{ }^{2} \operatorname{Var}\left(\eta_{\mathrm{ij}}\right)$ see equation $7.5 \mathrm{in}[5]$

The overall mean square error of prediction is obtained by

$\operatorname{MSEP}\left(\sum \hat{\mathrm{C}}_{\mathrm{ij}}\right) \approx \sum \varphi_{\mathrm{j}} \mu_{\mathrm{ij}}{ }^{\delta}+\sum \mu_{\mathrm{ij}}^{2} \operatorname{Var}\left(\eta_{\mathrm{ij}}\right)+2 \sum \operatorname{Cov}\left(\eta_{\mathrm{ij}} \eta_{\mathrm{ik}}\right) \mu_{\mathrm{ij}} \mu_{\mathrm{ik}}$

Table 1 reports the mean ultimate loss reserve (column 2), outstanding loss reserve (column 3 ), ultimate prediction uncertainty (column 4), the coefficient of variation for outstanding loss (column 5) and single year claims development uncertainty (column 6) obtained by standard CL model.

In Table 1 it can be analyzed that CL model estimates the claims uncertainty for the ultimate run-off (See Column 4), as well as the single year, measure by single year CDR(see Column 6). However, it does not generate the 99.5 percentile confidence interval for the single year. This issue is also highlighted in [8]. It can be resolved by incorporating bootstrapping methodology to obtain the empirical distribution of CDR then the 99.5 percentile requirement could be fulfilled.

\section{Parametric Bootstrapping}

The more comprehensive algorithmic version of bootstrap is given by Efron and Tibshirani [15]. It is not a model but a method to generate a sampling distribution of data by generating pseudo samples. Those are obtained by random selection from observed data. This is only possible if the data is independent and identically distributed and is exactly fitted to the underlying model. The bootstrapping approach is used to estimate uncertainty using prediction errors and is an applicable method under Solvency II regime. This method provides full empirical distribution of one-year outstanding loss that is the distribution of CDR.

The bootstrap algorithm executes two-level simulation approach. In level one a regular CL model is applied to the paid or incurred claims run-off trapezoid. This produces the scaled Pearson residuals and then has to bootstrap it $\mathrm{N}$ number of times to estimate incremental payments with the CL model. In the next level, the simulation of the process error takes place with the bootstrap value as the mean and incorporating the assumed process distribution. This study uses two different process distributions namely gamma distribution and over-dispersed Poisson distribution.

Following [5] to linearise the multiplicative model a logarithmic link function is assumed

$$
\begin{aligned}
& \log E\left(C_{i j}\right)=\log \left(\mu_{i j}\right)=\mu+\alpha_{i}+\beta_{j}=\eta_{i j} \\
& \hat{C}_{i j}=\exp \left(\hat{\eta}_{i j}\right)
\end{aligned}
$$

The general form of the over-dispersed-Poisson distribution is:

$$
\begin{aligned}
& \mathrm{E}\left(\mathrm{C}_{\mathrm{ij}}\right)=\mu_{\mathrm{ij}}=\alpha_{\mathrm{i}} \beta_{j} \\
& \operatorname{Var}\left(\mathrm{C}_{\mathrm{ij}}\right)=\varphi \mathrm{E}\left(\mathrm{C}_{\mathrm{ij}}\right)=\varphi \alpha_{\mathrm{i}} \beta_{\mathrm{j}}=\varphi \mu_{\mathrm{ij}}
\end{aligned}
$$

The $\varphi$ is unknown dispersion parameter that is estimated from the data.

The general form of the gamma distribution is:

$$
\mathrm{E}\left(\mathrm{C}_{\mathrm{ij}}\right)=\mu_{\mathrm{ij}}=\alpha_{\mathrm{i}} \beta_{\mathrm{j}}
$$

$\operatorname{Var}\left(\mathrm{C}_{\mathrm{ij}}\right)=\varphi\left(\mathrm{E}\left(\mathrm{C}_{\mathrm{ij}}\right)\right)^{2}=\varphi \mu_{\mathrm{ij}}^{2}$

The mean square error of prediction for individual cell is obtained by

See equation 7.9 in [5]

The following bootstrapping algorithm relies on $[3,4,5,9,16$, 17].

\subsection{The Bootstrap Algorithm}

Level one

Estimate the parameters $\hat{\mu},\left(\hat{\alpha}_{\mathrm{i}}\right)_{1 \leq \mathrm{i} \leq \mathrm{I}}$ and $\left(\hat{\beta}_{\mathrm{j}}\right)_{1 \leq \mathrm{j} \leq \mathrm{J}}$ from observed cumulative data $\left(\mu_{\mathrm{ij}}\right)_{1 \leq i+j \leq \mathrm{I}}$

Fit chain ladder model to the observed data and predict the claims $\left(\hat{\mu}_{\mathrm{ij}}\right)_{1 \leq \mathrm{i}+\mathrm{j} \leq \mathrm{I}}$ per origin year to fill the lower trapezoid shown in figure 1 by

Evaluate the best estimate at I, BE $\mathrm{I}=\sum_{\mathrm{i}+\mathrm{j} \geq \mathrm{I}+1} \exp \left(\hat{\eta}_{\mathrm{ij}}\right)$

Calculate dispersion parameter $\hat{\varphi}=\frac{\sum\left(\mathrm{r}_{\mathrm{ij}}^{\mathrm{p}}\right)^{2}}{\mathrm{n}-\mathrm{p}}$

Here $\frac{\sum\left(\mathrm{r}_{\mathrm{ij}}\right)^{2}}{\mathrm{n}-\mathrm{p}}$ is Pearson residual of $\mathrm{X}_{\mathrm{ij}}$ and these residuals are adjusted by factor $\sqrt{\frac{n}{n-p}}$

I. Calculate the scaled Pearson residual by

$$
r_{i j}^{p}=\frac{C_{i j}-\hat{\mu}_{i j}}{\sqrt{V\left(\hat{\mu}_{i j}\right)}} \forall i, j: 1 \leq i+j \leq I-1
$$

$\mathrm{r}_{\mathrm{ij}}^{\mathrm{p}}=\frac{\mathrm{C}_{\mathrm{ij}}-\hat{\mu}_{\mathrm{ij}}}{\sqrt{\hat{\varphi} \hat{\mu}_{\mathrm{i} j}{ }^{\delta}}} \forall \mathrm{i}, \mathrm{j}: 1 \leq \mathrm{i}+\mathrm{j} \leq \mathrm{I}-1$

Where $\hat{\varphi}=\delta=1$ for over dispersed Poisson and $\delta=2$ for gamma Level two: Bootstrap loop

Resample residuals $\stackrel{\mathrm{A}}{\mathrm{r}_{\mathrm{ij}}}$ with replacement and construct pseudo

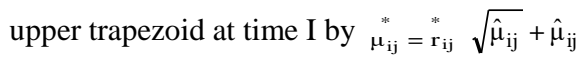

Restimate the model and obtain new parameters with this pseudodata $\hat{\mu}_{\mathrm{i}}{ }^{* 1}, \hat{\alpha}_{\mathrm{i}}{ }^{* 1}$ and $\hat{\beta}_{\mathrm{j}}{ }^{* 1}$

Obtain the expected values $\left(\hat{\mu}_{\mathrm{ij}}{ }^{* 1}\right)_{\mathrm{i}+\mathrm{j}=\mathrm{I}+1}$ in the sub-diagonal.

In order to take into account, the process errors simulate the empirical distribution with mean $\hat{\mu}_{\mathrm{ij}}{ }^{* 1}$ and variance $\hat{\varphi} \hat{\mu}_{\mathrm{ij}}{ }^{\delta}$ to produce the incremental payments $\mu_{\mathrm{ij}}{ }^{*}$. The single accounting year payments are $\mathrm{F}_{\mathrm{i}+1}^{*}=\sum_{\mathrm{i}+\mathrm{j}=\mathrm{I}+1} \mathrm{X}_{\mathrm{ij}}{ }^{*}$

Re-estimate model parameters in order to obtain new expected value $\left(\mu_{\mathrm{ij}}^{* 2}\right)_{\mathrm{i}+\mathrm{j} \geq \mathrm{I}+2}$

Calculate the best estimate at time $\mathrm{I}+1$ by $\mathrm{BE}_{\mathrm{I}+1}^{*}=\sum \sum \hat{\mu}_{\mathrm{ij}}{ }^{* 2}$

Obtain empirical distribution of CDR by $\mathrm{CDR}^{*}=\mathrm{F}_{\mathrm{I}+1}^{*}+\mathrm{BE}_{\mathrm{I}+1}^{*}-\mathrm{BE}_{\mathrm{I}}$

End of iteration *1

Return to level two until the thousand iterations 


\section{Results}

The bootstrap algorithm is applied to the data obtained from [13]. Table 2 and Table 3 reflect the results obtained through the bootstrap algorithm assuming the gamma distribution and overdispersed Poisson distribution respectively.

Table 2: Reserve estimation using gamma distribution

\begin{tabular}{|c|c|c|c|c|}
\hline Year & $\begin{array}{c}\text { Mean } \\
\text { Ultimate }\end{array}$ & $\begin{array}{c}\text { Mean } \\
\text { IBNR }\end{array}$ & $\begin{array}{c}\text { IBNR } \\
\text { msep }^{1 / 2} \\
99.5 \%\end{array}$ & $\begin{array}{c}\text { CDR(1) } \\
\text { msep }^{1 / 2} \\
99.5 \%\end{array}$ \\
\hline 1 & 7,518 & 0.0 & 0.0 & 0.0 \\
\hline 2 & 9,468 & 13.1 & 285.3 & 285.3 \\
\hline 3 & 13,220 & 58.9 & 398.3 & 401.0 \\
\hline 4 & 9,244 & 141.0 & 499.5 & 464.0 \\
\hline 5 & 7,804 & 217.7 & 717.0 & 547.7 \\
\hline 6 & 7,732 & 397.0 & 954.9 & 817.1 \\
\hline 7 & 8,606 & 815.1 & $1,597.3$ & $1,407.1$ \\
\hline 8 & 11,071 & $1,819.9$ & $3,014.7$ & $2,703.3$ \\
\hline 9 & 10,069 & $4,007.2$ & $5,891.3$ & $5,704.1$ \\
\hline 10 & 9,913 & $8,830.2$ & $16,476.1$ & $15,891.0$ \\
\hline Total & 94,645 & 16,300 & 24740.9 & 24256.7 \\
\hline
\end{tabular}

Table-2 reports the estimated mean ultimate claims amount, mean outstanding loss, the prediction uncertainty of outstanding loss, the quantiles of outstanding loss and claims development result obtained by using gamma bootstrap.

Table 3: Reserve estimation using over-dispersed Poisson distribution

Table 3: Reserve estimation using over-dispersed Poisson distribution
\begin{tabular}{|c|c|c|c|c|}
\hline Year & $\begin{array}{c}\text { Mean } \\
\text { Ultimate }\end{array}$ & $\begin{array}{c}\text { Mean } \\
\text { IBNR }\end{array}$ & $\begin{array}{c}\text { IBNR } \\
\text { msep }^{1 / 2} \\
99.5 \%\end{array}$ & $\begin{array}{c}\text { CDR(1) } \\
\text { msep }^{1 / 2} \\
99.5 \%\end{array}$ \\
\hline 1 & 7,518 & 0 & 0.0 & 0.0 \\
\hline 2 & 9,469 & 13.9 & 269.29 & 269.3 \\
\hline 3 & 13,221 & 60.3 & 377.2 & 384.4 \\
\hline 4 & 9,242 & 138.5 & 551.2 & 505.7 \\
\hline 5 & 7,804 & 218.1 & 760.2 & 603.0 \\
\hline 6 & 7,735 & 400.4 & 942.8 & 764.4 \\
\hline 7 & 8,597 & 806.3 & $1,584.5$ & $1,366.0$ \\
\hline 8 & 11,091 & $1,840.1$ & $3,114.5$ & $2,672.8$ \\
\hline 9 & 10,111 & $4,048.9$ & $6,061.0$ & $5,886.8$ \\
\hline 10 & 9,772 & 8.689 .2 & $15,463.9$ & $15,537.9$ \\
\hline Total & 94,560 & 16,216 & 23378.0 & 23405.3 \\
\hline
\end{tabular}

Table 3 reports the estimated mean ultimate claims amount, mean outstanding loss, the prediction uncertainty of outstanding loss, the quantiles of the prediction uncertainty in outstanding loss and claims development result obtained by using over-dispersed Poisson distribution in bootstrap.

The comparison between result reported in Table- 2 and Table- 3 is carried out to compare the behavior of estimation predicted by assuming two different process distributions in the bootstrap algorithm. Both fitted distributions provide similar results but not identical, the ultimate loss (Mean Ultimate), outstanding loss(Mean IBNR), mean square errors of outstanding loss(IBNR S.E.) are not very different. However are greater in gamma bootstrap than in Poisson bootstrap. The quantiles of the CDR and IBNR at $99.5 \%$ in gamma bootstrap are greater than Poisson bootstrap. It indicates that prediction uncertainty is higher in gamma bootstrap as compared to Poisson bootstrap.

Finally, by analyzing this comparison, two conclusions are extracted.

1. The gamma bootstrap prediction errors are little higher than the prediction errors in over-dispersed Poisson bootstrap.

2. The values of the quantiles in gamma bootstrap are higher than over-dispersed-Poisson bootstrap.

Mostly the non- negative valued probability distributions are used in the loss reserving by the actuaries. The log-normal distribution is used widely in the loss reserving literature. Because in other distributions the pseudo data negative values have to be replaced by the zeros in practice. However log-normal generate higher prediction errors than gamma and over-dispersed Poisson because of the conservative characteristics.

\section{Conclusion}

This paper studies the reserve estimation requirements under the solvency-II regime, especially the value-at-risk measure with 99.5 percentile confidence level over the 12 months period requirement. In this connection, CDR concept is incorporated into the chain ladder model to estimate the loss reserve over a one-year period. However, this method does not generate the 99.5 percentile (quantiles). Further, the bootstrap methodology is applied to obtain the distribution for the CDR from which the means and quantiles of the IBNR and CDR are obtained. In the bootstrap algorithm, two different process distributions are used namely gamma and overdispersed Poisson distribution to generate the distribution of reserve.

Finally, a comparison is being carried out between gamma and over-dispersed Poisson bootstrap results and it is found that both distributions generate similar predictions but not identical. In fact, the over-dispersed Poisson distribution produces less estimation errors than the gamma distribution.

\section{References}

[1] Solvency-II Directive 2009/138/EC, Official Journal of the European Union, retrieved on September 2016 , fromhttp://eurlex.europa.eu/LexUriServ/LexUriServ.do?uri=OJ:L:2 009:335:0001:0155:en:PDF

[2] EIOPA (European Insurance and Occupational Pensions Authority) Revised Technical Specifications for the Solvency-II valuation and Solvency Capital Requirements calculations (Part I), retrieved on August 2016, from https://eiopa.europa.eu/Publications/Standards/A_Revised_Techni cal_Specifications_for_the_Solvency_II_valution_and_Solvency_C apital_Requirements_calculations_Part_I_pdf

[3] M. Merz and M.V. Wüthrich (2008), Modelling the claims development result for solvency purpose, Casualty Actuarial Society EForum, retrived on August 2016 from https://www.casact.org/pubs/forum/08fforum/21Merz_Wuetrich.pd $\mathrm{f}$

[4] T. Mack (1993), Distribution-free calculation of the standard error of chain ladder reserves estimates, Astin Bulletin, Vol. 23, No. 2, pp. 213-225.

[5] P. D. England and R. J. Verrall (2002), Stochastic claims Reserving in General Insurance (with discussion), British Actuarial Journal, Vol. 8, No. 3, pp. 443-554. doi:10.1017/S1357321700003809

[6] T. Mack, G. Quarg and C. Braun (2006), The mean square error prediction in the chain ladder reserving -A comment, Astin Bulletin, Vol. 36, No. 2, pp. 543-552. doi:10.1017/S051503610001463X

[7] M. Buchwalder, H. Bühlmann, M. Merz and M.V. Wüthrich (2006), The mean square error of prediction in the chain ladder reserving method (Mack and Murphy revisited), ASTIN Bulletin,Vol.36, No. 2 ,pp. 521-542, doi:10.1017/S0515036100014628

[8] Robin (2012), A Practical Way to Estimate One-year Reserve Risk. Casualty Actuarial Society E-Forum, retrived on August 2016 from https://www.casact.org/pubs/forum/12sumforum/Robbin.pdf

[9] P. J. R. Pinheiro, João Manuel Andrade e Silva, \& Maria de Lourdes Centeno (2003), Bootstrap Methodology in Claim Reserving, The Journal of Risk and Insurance, Vol. 70, No. 4, pp. 701714. doi:10.1046/j.0022-4367.2003.00071.x

[10] E. Ohlsson, and J. Lauzeningks, The one-year non-life insurance risk. ASTIN Colloquium 2008, Manchester.

[11] B. Efron (1979), Bootstrap Methods: Another Look at the Jackknife, The Annals of Statistics, Vol. 7, No.1, pp.1-26.

[12] F. De Felice and Moriconi (2006), Process error and estimation error of year-end reserve estimation in the distribution free chainladder model, Alef Working Paper, Rome.

[13] H. Liu and R. Verrall (2010), Bootstrap estimation of the predictive distributions of reserves using paid and incurred claims, Variance, Vol. 4, No. 2, pp. 121-135. 
[14] T. Mack (1994), Which stochastic model is underlying the chain ladder method. Insurance: Mathematics and Economics, Vol. 15, No.1 , pp. 133-138.

[15] B. Efron and R. J. Tibshirani (1993), An introduction to the bootstrap. Chapman and Hall, New York.

[16] P.D. England and R. J. Verall (1999), Analytic and bootstrap estimates of prediction errors in claim reserving, Insurance: Mathematics and Economics, Vol. 25,No. 3 , pp. 281-293. https://doi.org/10.1016/S0167-6687(99)00016-5

[17] P.D. England and R. J. Verall (2006), Predictive distribution of outstanding liabilities in general insurance, Annals of Actuarial Science, Vol. 1, No. 2 , pp. 221-270. doi:10.1017/S1748499500000142 